\title{
Lessons of the month 1: Fatal oesophago-pericardial fistula with cerebral air embolism after elective atrial fibrillation ablation
}

\author{
Authors: Jonathan Dutton, ${ }^{\mathrm{A}}$ Marco Morosin, ${ }^{\mathrm{B}}$ Rita Fernandez-Garda, ${ }^{\mathrm{C}}$ Vladamir Anikin, ${ }^{\mathrm{D}}$ Ana Hurtado-Doce ${ }^{\mathrm{E}}$ \\ and Nicholas Lees ${ }^{\mathrm{F}}$
}

\section{Oesophago-pericardial fistula following any} electrophysiological procedure is a rare, and potentially, life-threatening condition. Initial presentation can easily be misdiagnosed, as symptoms vary and are not specific. Echocardiography is an invaluable tool to diagnose and rule out complications. We present the case of a 68-yearold patient who developed an oesophago-pericardial fistula complicated with purulent pericarditis, sepsis and cerebral air embolism. In conclusion, this case report encourages physicians to use strategies that may help with early diagnosis and lead to potential lifesaving interventions.

KEYWORDS: Cardiology, atrial fibrillation, ablation

\section{Case presentation}

A 62-year-old man presented with progressively worsening dyspnoea. Two weeks earlier he underwent elective pulmonary vein isolation with radiofrequency (RF) ablation for permanent atrial fibrillation (AF). He was anticoagulated with a direct factor-Xa inhibitor (rivaroxaban) immediately post-procedure and remained so when he presented to us.

His presenting rhythm was AF with an inferolateral ST-elevation. A myocardial infarction was suspected and urgent angiography was performed, which revealed no significant coronary occlusion. A bedside echocardiogram showed a $30 \mathrm{~mm}$ posterior pericardial effusion along the left ventricle border without signs of cardiac tamponade. A computed tomography (CT) thorax demonstrated

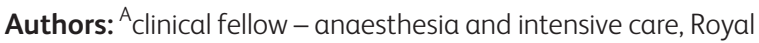
Brompton and Harefield NHS Foundation Trust, London, UK; ${ }^{\text {B }}$ clinical fellow - anaesthesia and intensive care, Royal Brompton and

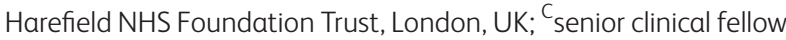
- anaesthesia and intensive care, Royal Brompton and Harefield


Royal Brompton and Harefield NHS Foundation Trust, London, UK; intensive care consultant, Royal Brompton and Harefield NHS Foundation Trust, London, UK; F consultant anaesthetist, Royal Brompton and Harefield NHS Foundation Trust, London, UK the presence of an isolated small gas collection at the level of the lower oesophagus with unclear intra- or extra-luminal location. The working diagnosis was pericarditis with associated reactive pericardial effusion; likely related to his recent RF ablation procedure. An ultrasound-guided pericardiocentesis was performed and $560 \mathrm{~mL}$ straw-coloured fluid aspirated, samples were sent to pathology.

Post-pericardiocentesis he developed a systemic inflammatory response, and empirical broad-spectrum antibiotic was commenced (piperacillin-tazobactam). Later group-A Streptococcus pyogenes was isolated from the pericardial aspirate and antibiotics were suitably rationalised as per antibiogram guidance.

After 3 days the patient improved significantly, and a repeated transthoracic echocardiogram showed a reduction in the pericardial effusion without re-accumulation of fluid; he was stable and therefore suitable for transfer to level-2 care.

Three days after his intensive therapy unit (ITU) discharge, he developed a dense left hemiplegia with facial drooping and hypotonia, all in keeping with a total anterior circulation stroke syndrome. After discussion with the local stroke specialist team, thrombolysis was felt to be contraindicated due to a high risk of bleeding.

Twenty-four hours later, he developed generalised tonic-clonic seizure activity requiring sedation, intubation and levetiracetam was commenced.

Despite optimal treatment he continued to deteriorate and a CT thorax and abdomen revealed a pneumopericardium, accumulation of a pericardial effusion, and a new left lower lobe consolidation; broad spectrum antibiotics were started (Fig 1a). Additionally, he was heparinised with unfractionated heparin as it was felt his ongoing risk of further thrombotic episodes outweighed the bleeding risks at this stage.

Within 72 hours of achieving therapeutic heparinisation he had an episode of melaena. An oesophago-gastroduodenoscopy (OGD) found the source to be a large oesophageal tear (Fig 1b) with direct visualisation of the left atrium. In order to better define extent of the injury, a CT thorax was repeated with enteral contrast injected through a proximal nasogastric tube. This demonstrated contrast extravasation anterior to the oesophagus at the level of the right inferior pulmonary vein (Fig 2a), tracking along the pericardium and surrounding the left atrium; 
Fig 1. (a) Intravenous contrast enhanced computed tomography thorax; demonstrating pneumopericardium. (b) Oesophago-gastroduodenoscopy: left atrium prompting into the oesophagus.
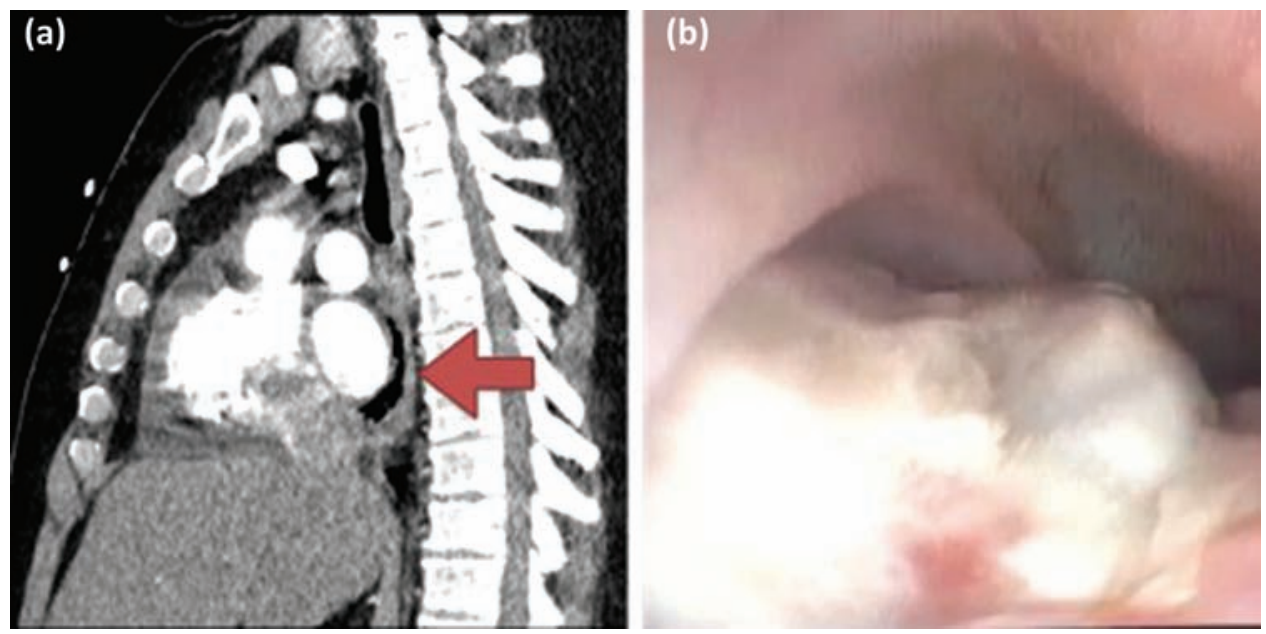

additionally, air bubbles were seen in the pericardial space. These findings were indicative of an oesophago-pericardial fistula.

At this point thoracic surgeons were involved in a

multidisciplinary approach, and he was taken to theatre.

They performed a jejunostomy and also placed a transgastric oesophageal drain. A tracheostomy was also performed in view of the likely expected prolonged ventilatory support.

In the following days, the oesophageal drain intermittently drained large volumes of blood requiring blood product transfusion. It was felt to be related to progression of his atriooesophageal fistula but, due to his poor general condition, it was decided to persist with conservative management.

The patient had a persistently low level of consciousness despite attempts to reduce sedation. An electroencephalogram (EEG) showed slow bioelectrical activity in the right hemisphere with response to painful stimuli but no signs of epileptiform status. A further CT brain revealed further ischaemic infarcts in the right frontal and parietal lobes with multiple foci of pneumocephalus at grey-white transition points; there were also brainstem and cerebellum infarcts (Fig 2b).
Given his catastrophic neurological prognosis, and in the setting of uncontrolled mixed hypovolemic and distributive shock with ongoing multi-organ failure, further intervention was felt to be futile and care was withdrawn.

\section{Discussion}

We report an uncommon, but important life-threatening complication of AF RF ablation, which is associated with very high morbidity and mortality. Given the findings on cross-sectional imaging, the intermittent haemorrhagic oesophageal drainage and the time-frame of the presentation (between 3 days and 5 weeks post ablation), we felt the most likely mechanism to contribute to the cerebral air embolism detected on CT brain was the presence of an atrial-oesophageal fistula. ${ }^{1}$ This patient had a typical presentation of this rare complication (sepsis, melaena, haematemesis, stroke and seizure). ${ }^{2,3}$

He had a history of Barrett's oesophagus in addition to multiple previous attempts of RF ablation procedures, both recognised as potential risk factors. Unfortunately, definite
Fig 2. (a) Computed tomography thorax with oral contrast: opacification of the oesophagus with focal extravasation at the level of the right inferior pulmonary vein. (b) Computed tomography head: ischaemic infarcts with air embolism at grey-white matter interface.
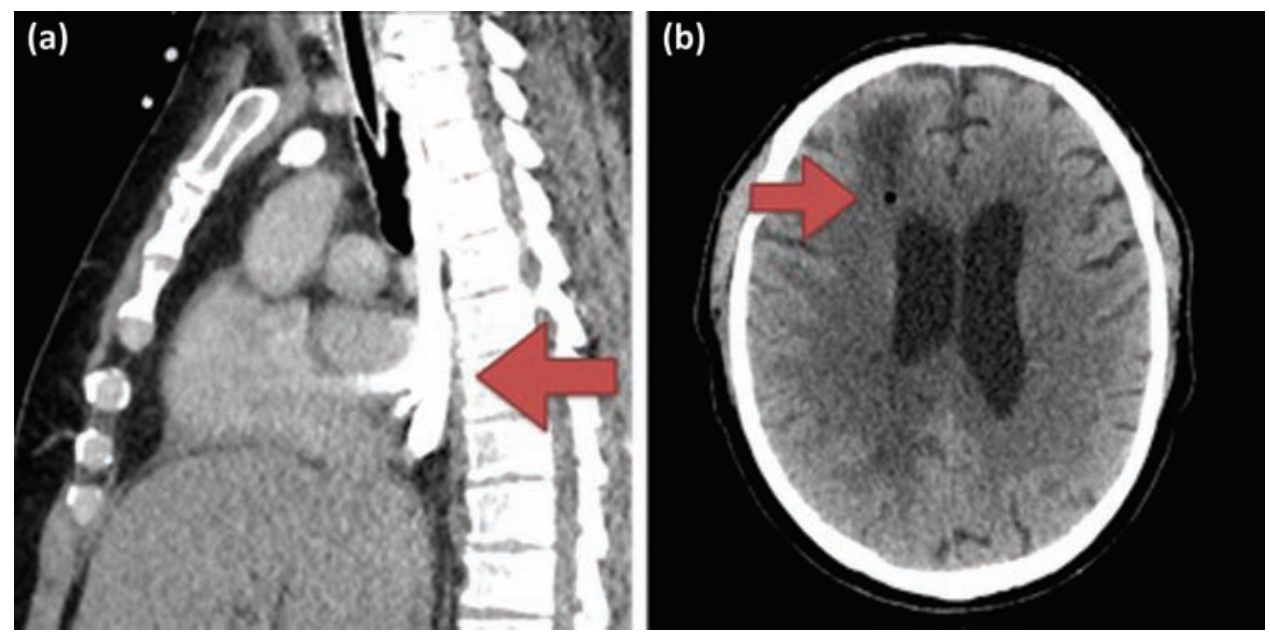
imaging was unsuitable for diagnosis, owing to his rapidly declining condition.

Treatment of atrio-oesophageal fistula requires prompt identification and emergent surgical intervention, for which high clinical suspicion is mandatory. Medical management alone and stent placement have been proved to be unsuccessful with nearly $100 \%$ mortality. ${ }^{4}$ In our case, haemodynamic instability and the devastating neurological prognosis precluded the option for definitive intervention.

Preventive strategies that have been investigated include oesophageal cooling, luminal oesophageal temperature monitoring and limiting RF power delivery on the mid-portion of posterior wall of left atrium. ${ }^{5}$ Despite preventative measures being taken, complications like that above can still occur with disastrous consequences for the patient.

\section{Conclusions}

Strategies to prevent oesophageal injury are commonly employed during RF ablation procedures, however, pericardial and oesophageal injuries are known and reported in the literature. Given the seriousness and poor prognosis of such complications, early recognition and intervention is important. .

\section{References}

1 Nair GM, Nery PB, Redpath C], Lam BK, Birnie DH. Atrioesophageal fistula in the era of atrial fibrillation ablation: a review. Can J Cardiol 2014:30:388e395.

2 Keshishian J, Young J, Hill E, Saloum Y, Brady PG. Esophageal injury following radiofrequency ablation for atrial fibrillation: injury classification. Gastroenterol Hepatol 2012;8:411-4.

3 Mohanty S, Santangeli P, Mohanty P et al. Outcomes of atrioesophageal fistula following catheter ablation of atrial fibrillation treated with surgical repair versus esophageal stenting. J Cardiovasc Electrophysiol 2014:25:579-84

4 Yousufa T, Keshmiria H, Bulwab Z et al. Management of atrio-esophageal fistula following left atrial ablation. Cardiol Res 2016;7:36-45.

5 Kapur S, Barbhaiya C, Deneke T, Michaud GF. Esophageal injury and atrioesophageal fistula caused by ablation for atrial fibrillation. Circulation 2017;136:1247-55

Address for correspondence: Dr Jonathan Dutton, Department of Anaesthesia and Critical Care, Royal Brompton and Harefield NHS Foundation Trust, Harefield Hospital, Hill End Road, Harefield, Uxbridge, London UB9 6JH, UK. Email: jonathan.dutton@doctors.org.uk

\section{Royal College} of Physicians

\section{Guidance on safe medical staffing}

The RCP's Guidance on safe medical staffing working party report aims to help those planning and organising core hospital medical services to answer the question: 'How many doctors or their alternatives, with what capabilities, do we need to provide safe, timely and effective care for patients with medical problems?'.

\section{Download the report at: www.rcplondon.ac.uk/safe-medical-staffing}
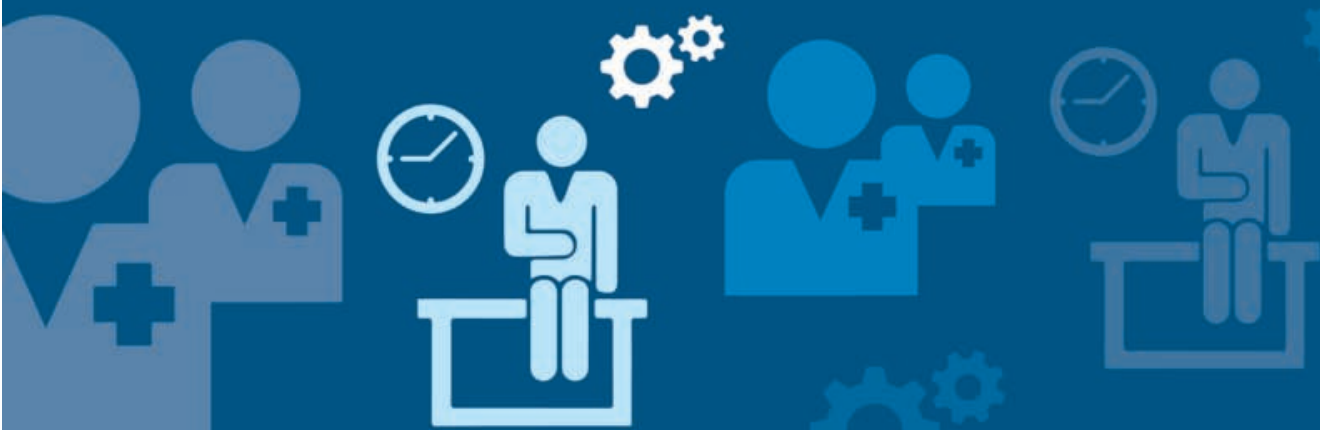